\title{
Florida FFA Vegetable Judging Competition: A Study Guide $^{1}$
}

\author{
Jennifer Lynn Gillett-Kaufman²
}

This guide links to articles about insects that are often used in the Florida FFA Vegetable Judging Competition. Click the name above each photo to learn more.

\section{Pests}

Aphids

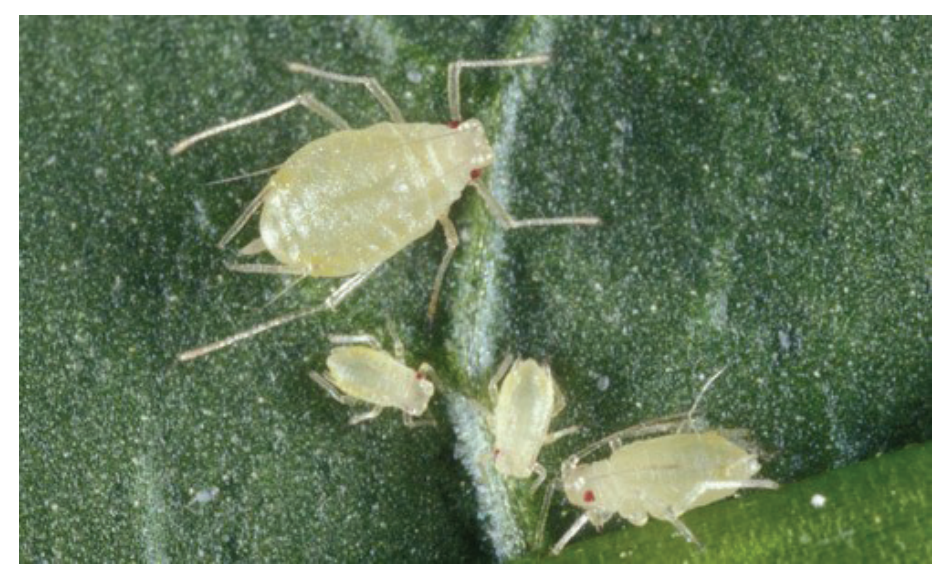

Figure 1. Female adult green peach aphids, Myzus persicae, with immatures.

Credits: USDA
Banded Cucumber Beetle

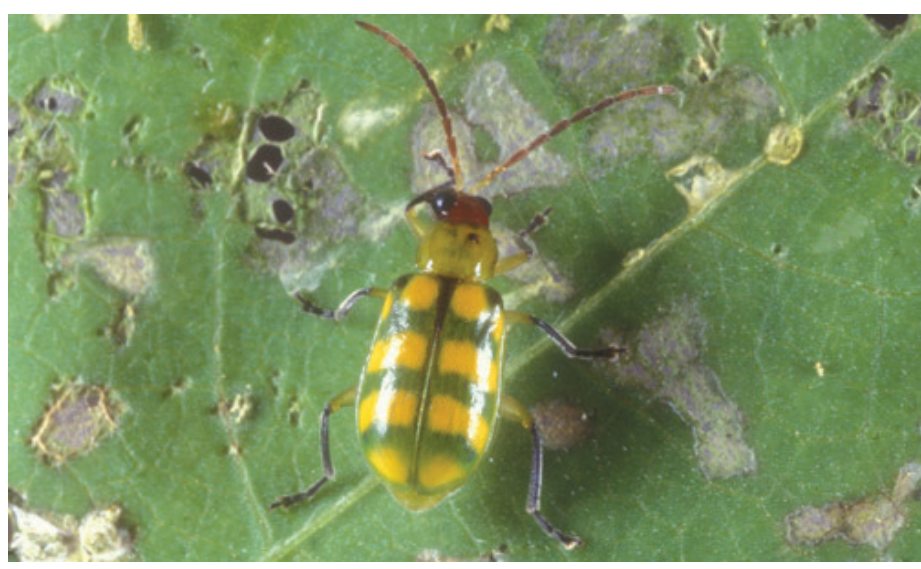

Figure 2. Adult banded cucumber beetle, Diabrotica balteata.

Credits: Jim Castner, UF/IFAS

Bean Leafroller

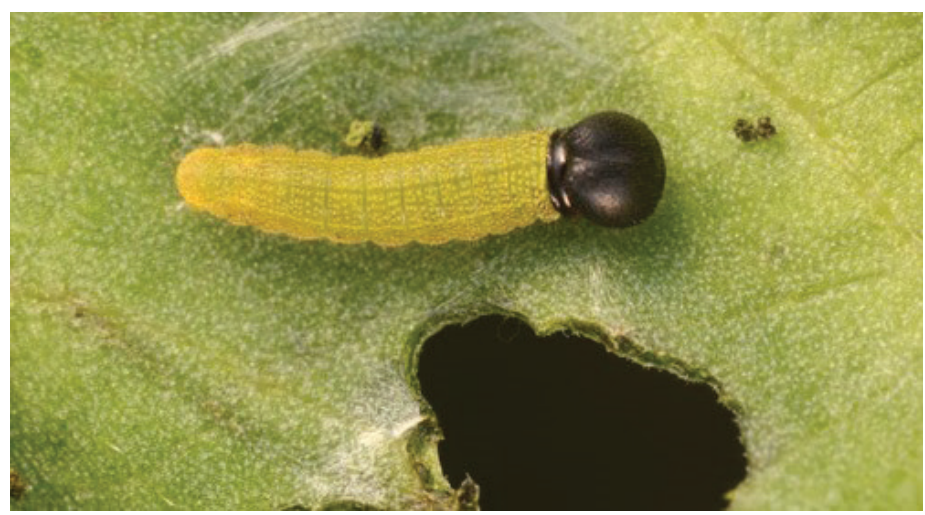

Figure 3. Larva of the bean leafroller, Urbanus proteus.

Credits: John L. Capinera, UF/IFAS

1. This document is 4HPSJ25, one of a series of the 4-H Youth Development Department, UF/IFAS Extension. Original publication date April 2018. Visit the EDIS website at http://edis.ifas.ufl.edu.

2. Jennifer Lynn Gillett-Kaufman, associate Extension scientist, Entomology and Nematology Department; UF/IFAS Extension, Gainesville, FL 32611.

The Institute of Food and Agricultural Sciences (IFAS) is an Equal Opportunity Institution authorized to provide research, educational information and other services only to individuals and institutions that function with non-discrimination with respect to race, creed, color, religion, age, disability, sex, sexual orientation, marital status, national origin, political opinions or affiliations. For more information on obtaining other UF/IFAS Extension publications, contact your county's UF/IFAS Extension office. 
Cabbage Looper

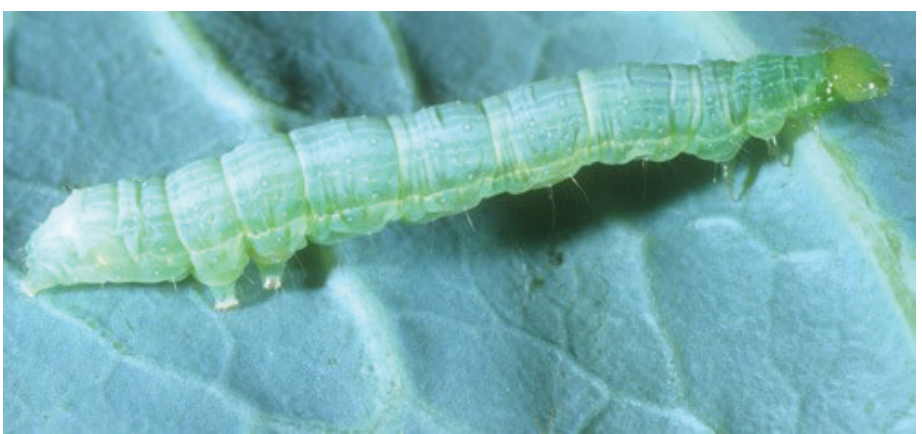

Figure 4. Early instar larva of the cabbage looper, Trichoplusia ni. Credits: John L. Capinera, UF/IFAS

Click Beetle

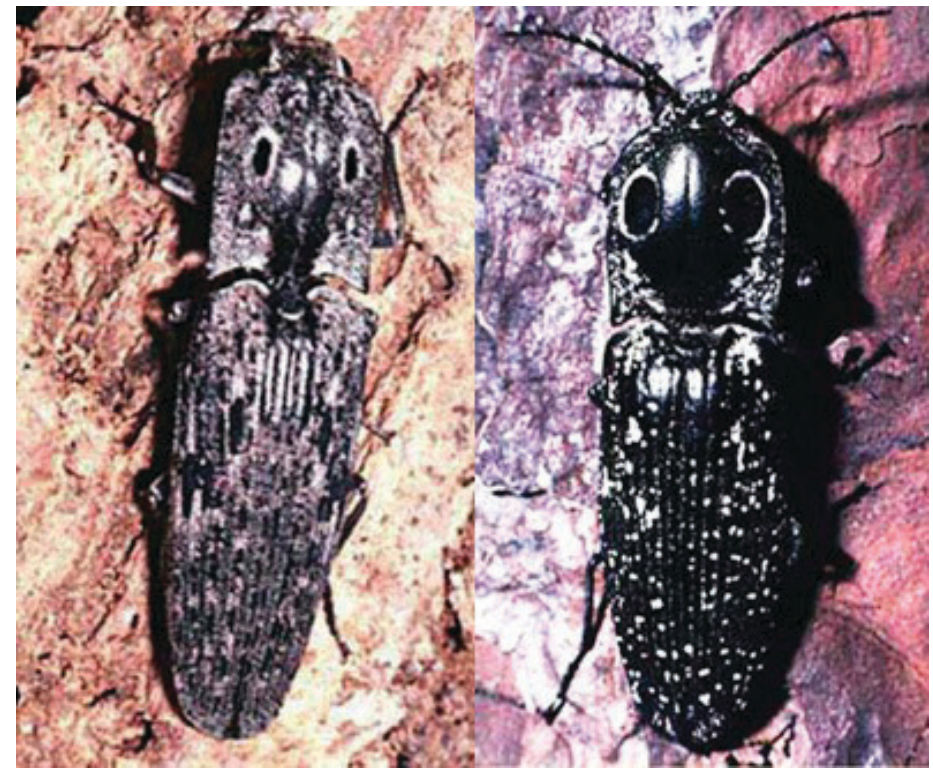

Figure 5. Adult click beetles: Alaus myops, left, and Alaus oculatus, right.

Credits: M. C. Thomas, Division of Plant Industry

Colorado Potato Beetle, Adult

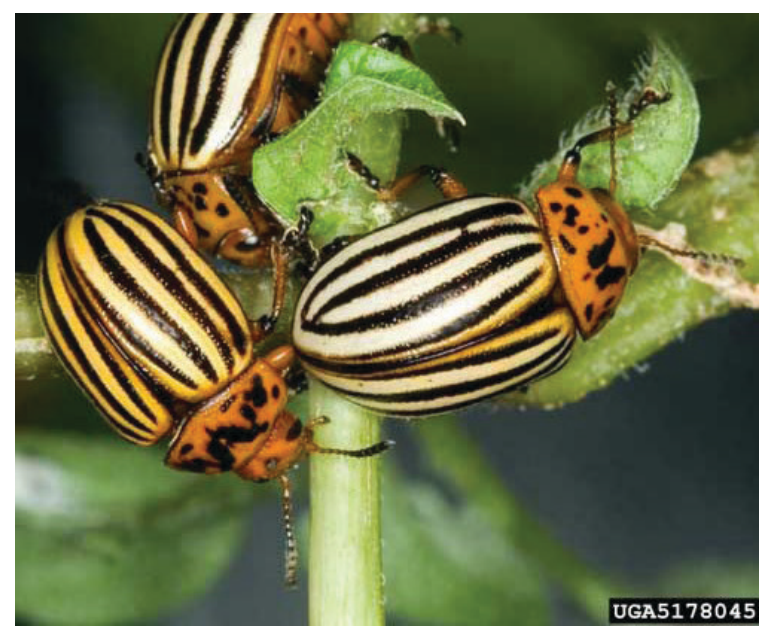

Figure 6. Colorado potato beetles, Leptinotarsa decemlineata, feeding on foliage.

Credits: David Cappaert, Michigan State University, www.

insectimages.org
Colorado Potato Beetle, Larva

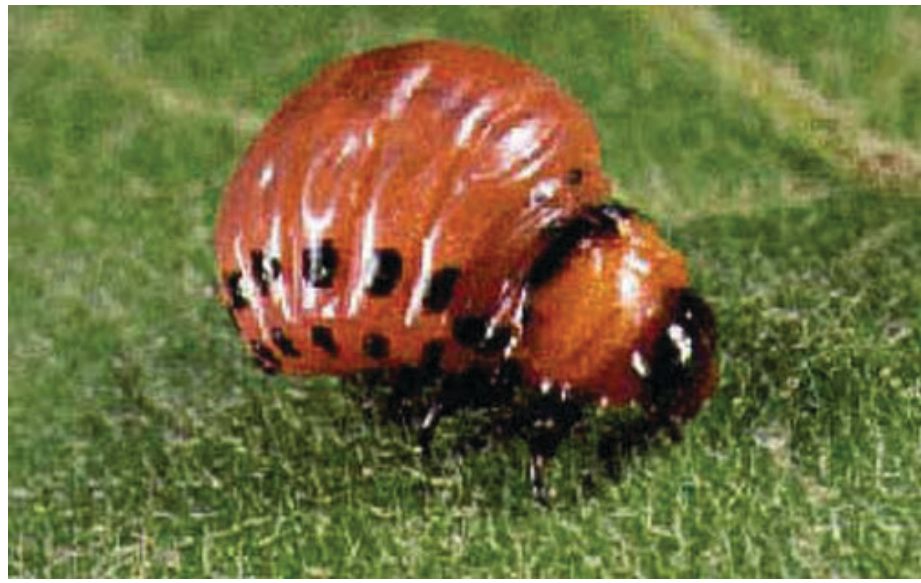

Figure 7. Larva of the Colorado potato beetle, Leptinotarsa decemlineata.

Credits: UF/IFAS

\section{Corn Earworm}

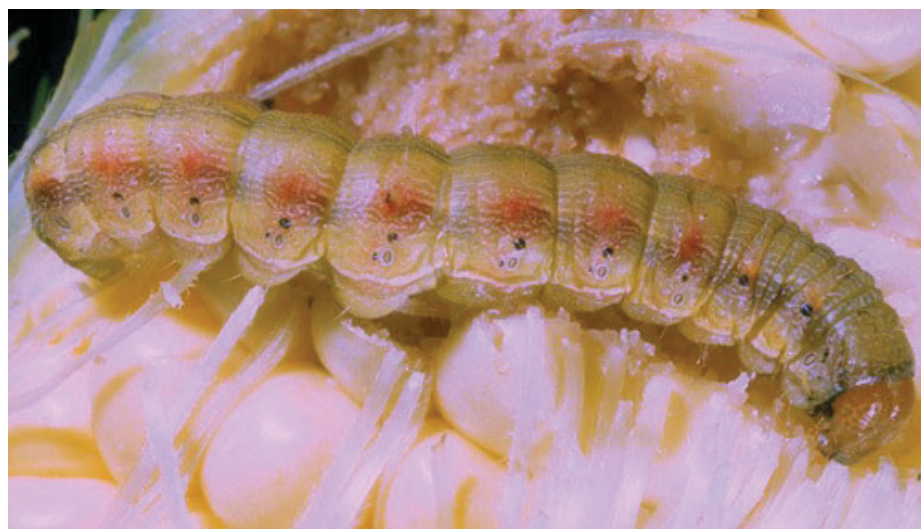

Figure 8. Larva of corn earworm, Helicoverpa zea. This is the lightcolored or greenish form.

Credits: John L. Capinera, UF/IFAS

\section{Cutworm}

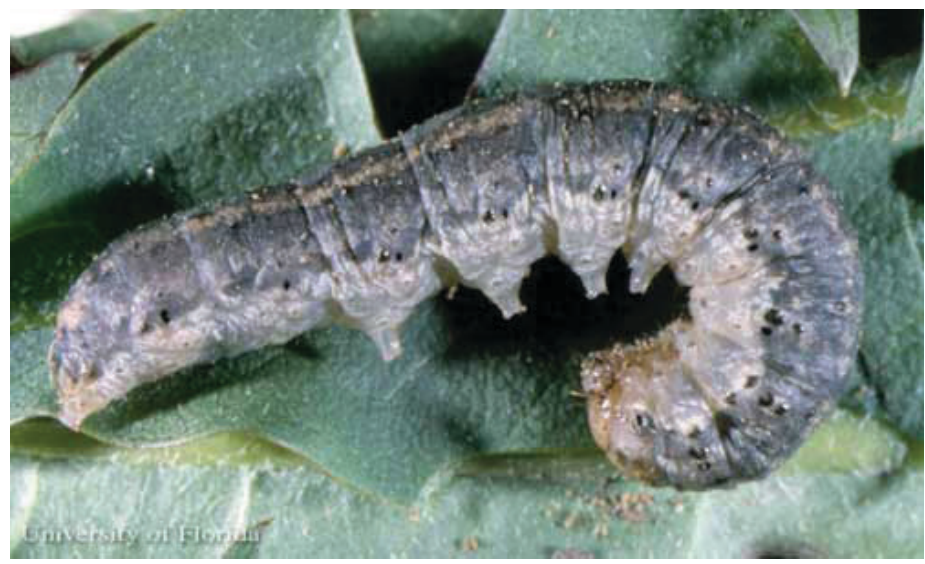

Figure 9. Side view of the larva of a black cutworm, Agrotis ipsilon. Credits: John L. Capinera, UF/IFAS 
Fall Armyworm

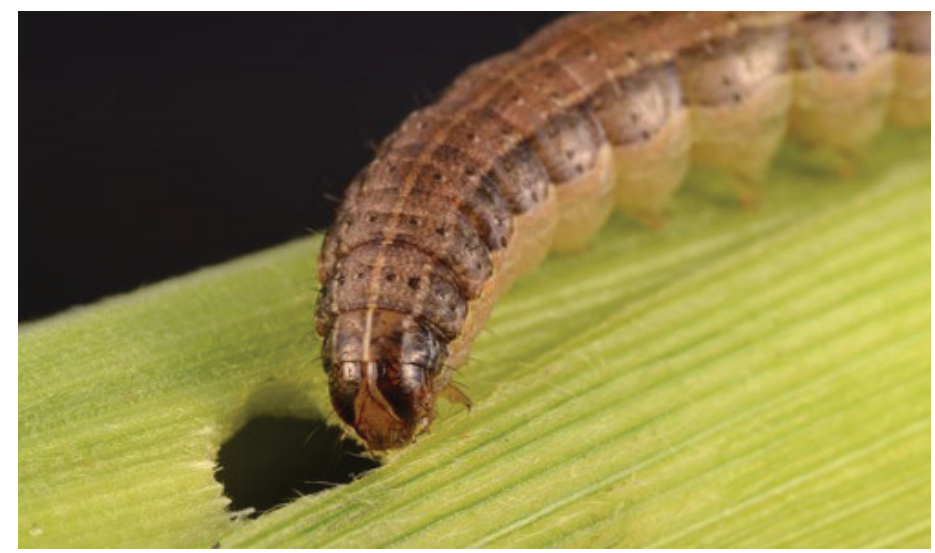

Figure 10. Larva of fall armyworm, Spodoptera frugiperda. Note the light-colored inverted " $Y$ " on the front of its head.

Credits: John L. Capinera, UF/IFAS

Field Cricket

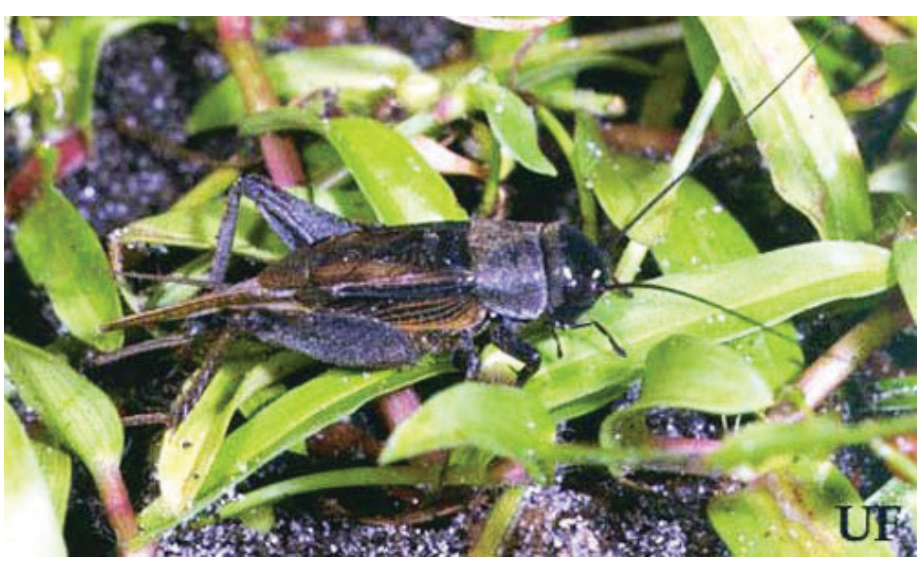

Figure 11. Long-winged, adult male southeastern field cricket, Gryllus rubens.

Credits: Paul M. Choate, UF/IFAS

${ }^{\star}$ Flea Beetle (links to BugGuide)

Green Stink Bug

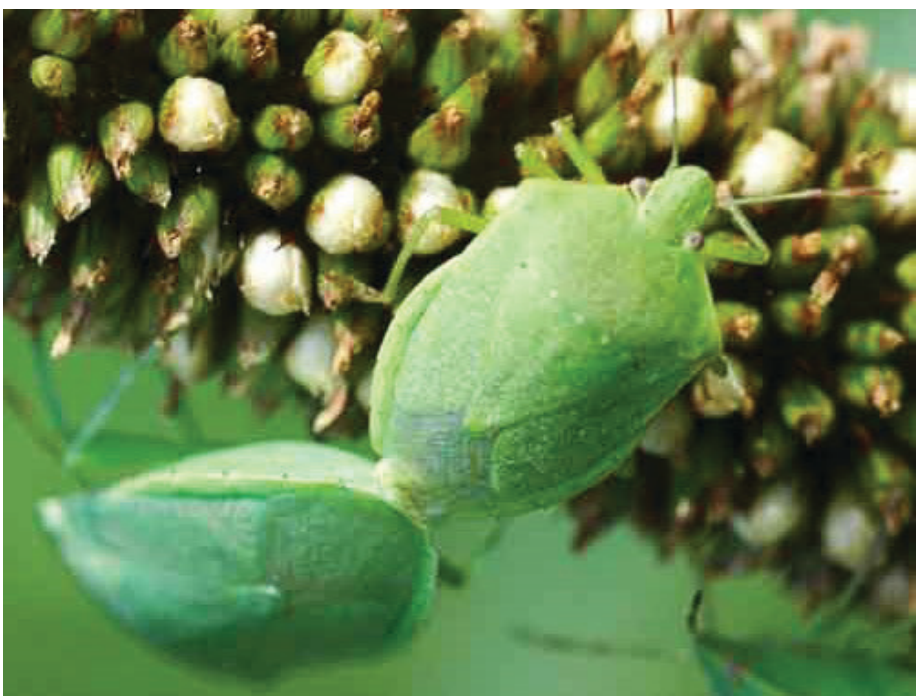

Figure 12. Adult green stink bugs, Chinavia halaris, on millet.

Credits: Russell F. Mizell III, UF/IFAS
Harlequin Bug

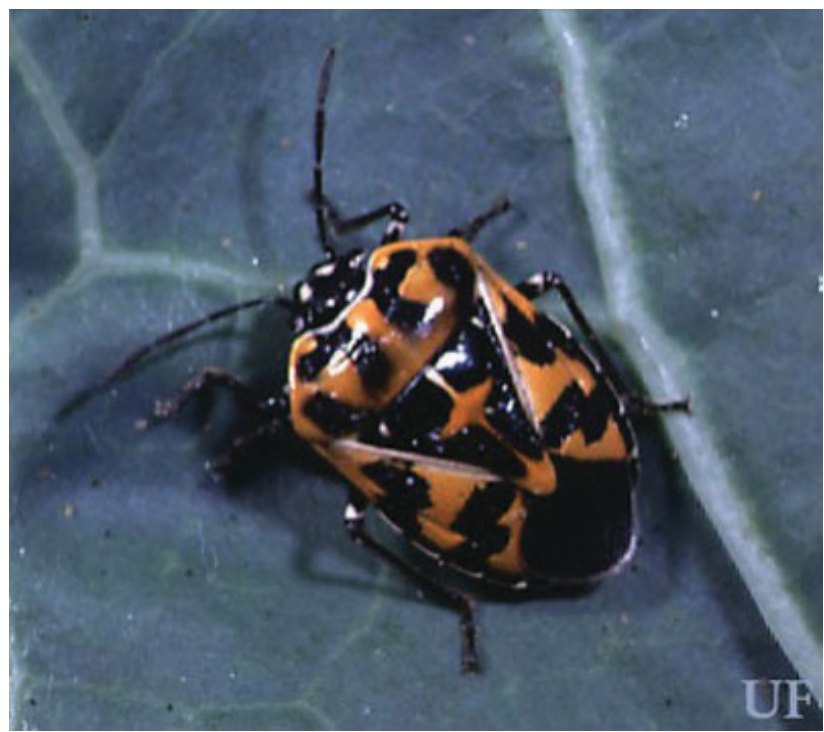

Figure 13. Adult harlequin bug, Murgantia histrionica.

Credits: James Castner, UF/IFAS

Imported Cabbageworm

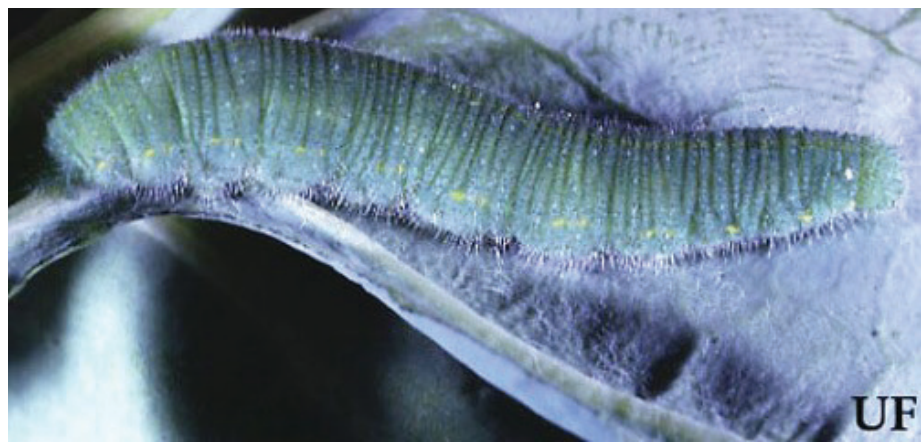

Figure 14. Larva of imported cabbageworm, Pieris rapae.

Credits: John L. Capinera, UF/IFAS

June Beetle

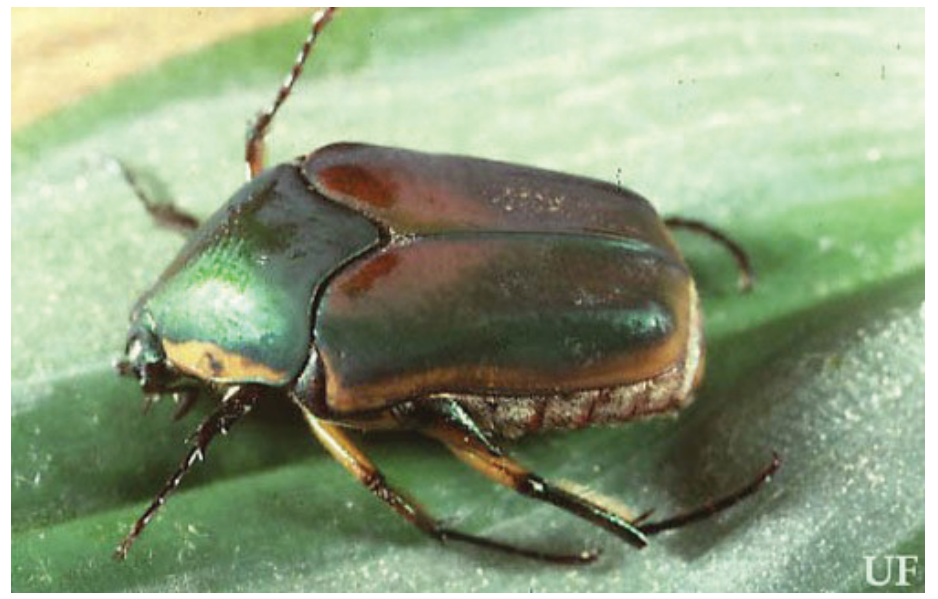

Figure 15. Adult green June beetle, Cotinis nitida.

Credits: John L. Capinera, UF/IFAS 
Leaf-Footed Plant Bug

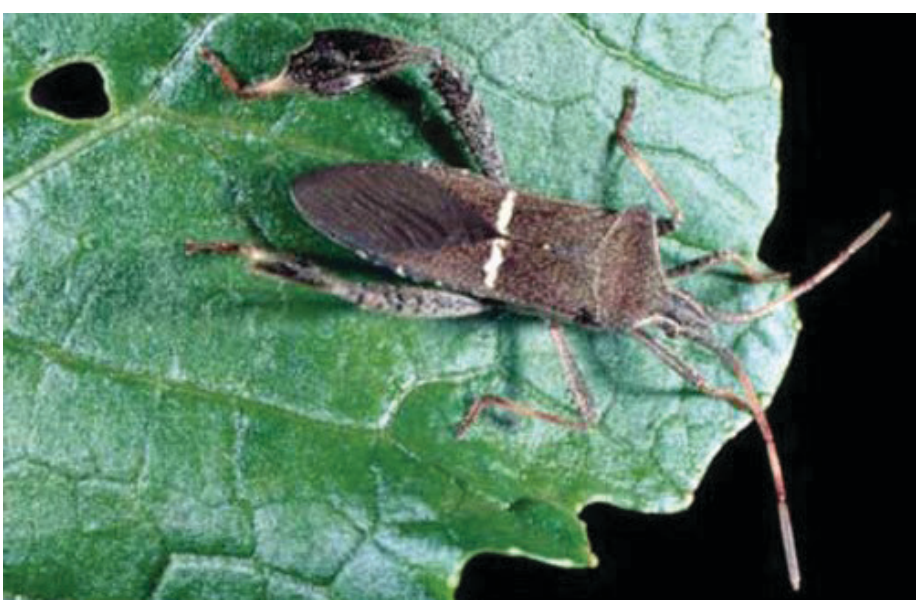

Figure 16. Adult leaf-footed bug, Leptoglossus phyllopus. Credits: John L. Capinera, UF/IFAS

Leafhopper

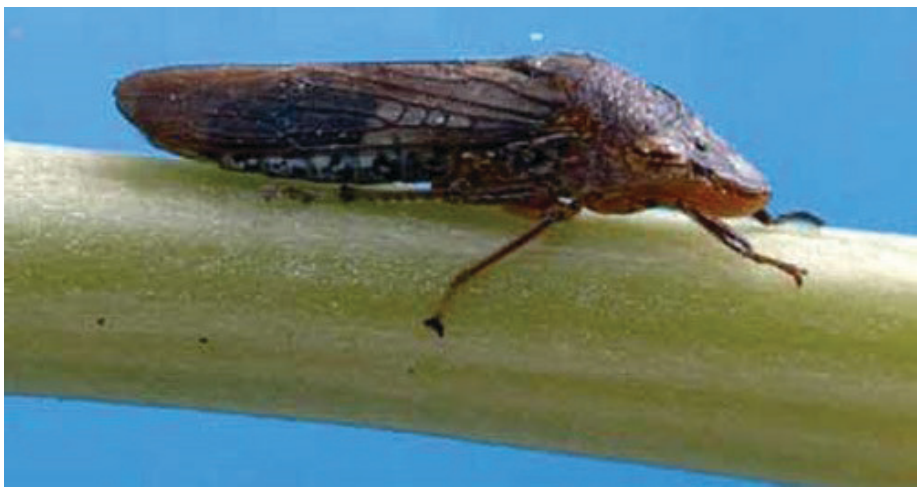

Figure 17. An adult Homalodisca vitripennis, the glassy-winged sharpshooter.

Credits: Chris Tipping, UF/IFAS

Leafminer

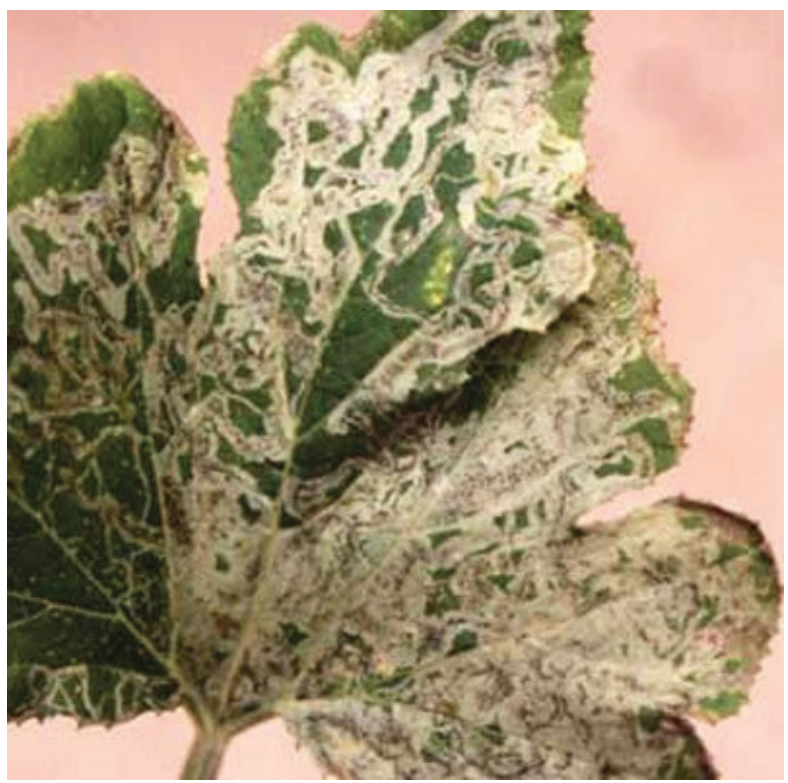

Figure 18. Mines in squash leaf caused by Liriomyza leafminers. Credits: James Castner, UF/IFAS
Lesser Cornstalk Borer

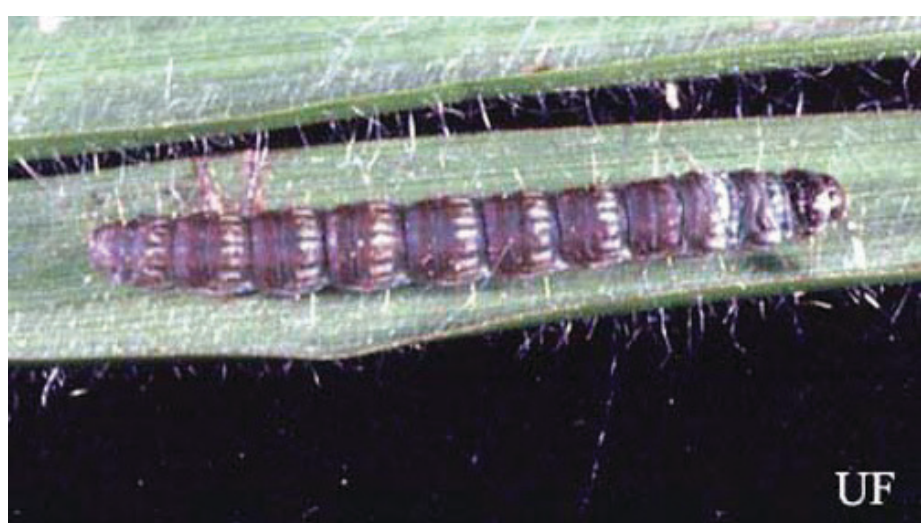

Figure 19. Mature larva of the lesser cornstalk borer, Elasmopalpus lignosellus.

Credits: John L. Capinera, UF/IFAS

Mexican Bean Beetle, Adult

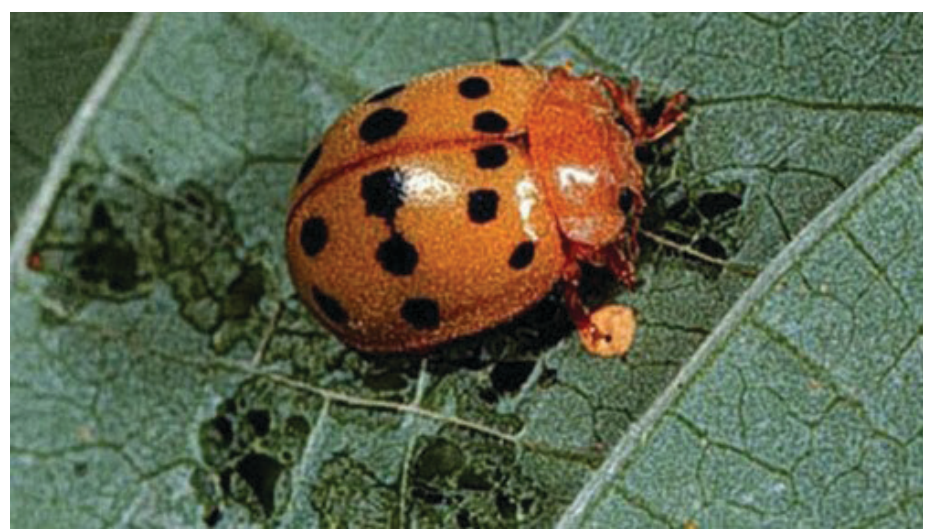

Figure 20. Adult Mexican bean beetle, Epilachna varivestis.

Credits: James Castner, UF/IFAS

Mexican Bean Beetle, Larva

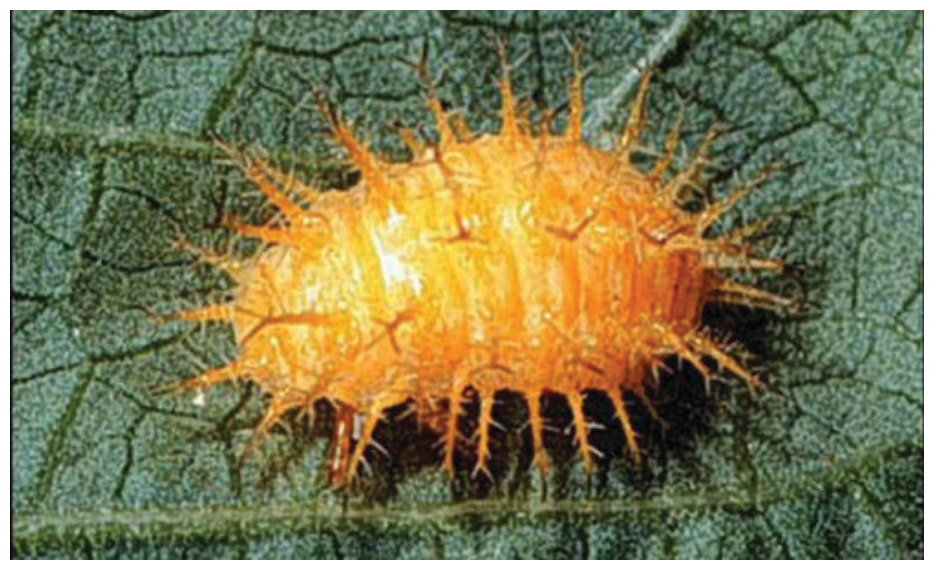

Figure 21. Larva of Mexican bean beetle, Epilachna varivestis. Credits: James Castner, UF/IFAS 
Mole Cricket

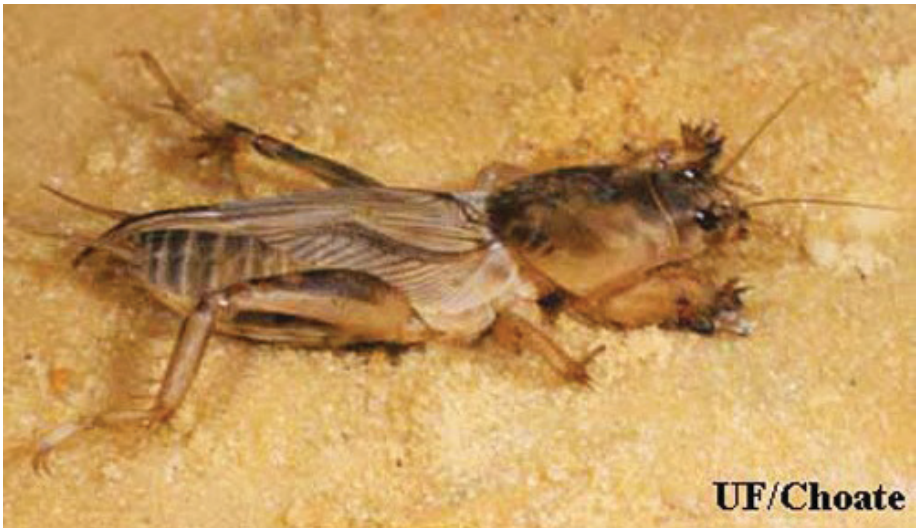

Figure 22. Adult tawny mole cricket, Neoscapteriscus vicinus.

Credits: Paul M. Choate, UF/IFAS

Pickleworm

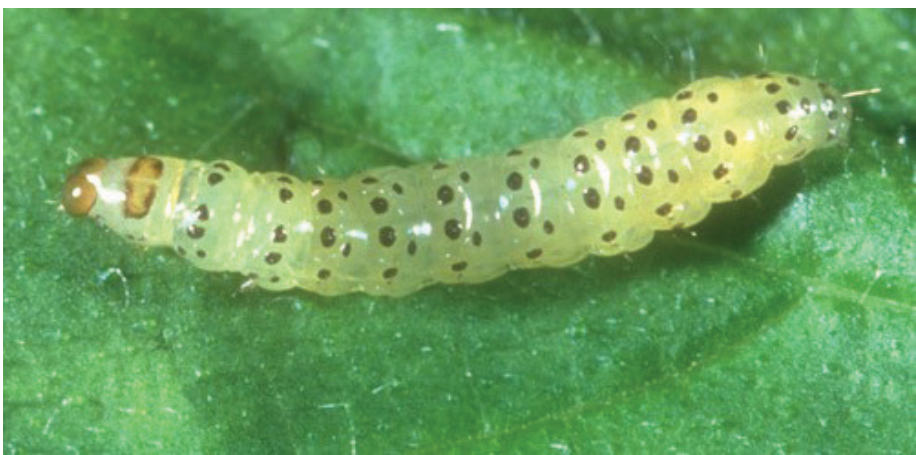

Figure 23. Young pickleworm larva, Diaphania nitidalis.

Credits: John L. Capinera, UF/IFAS

Squash Bug

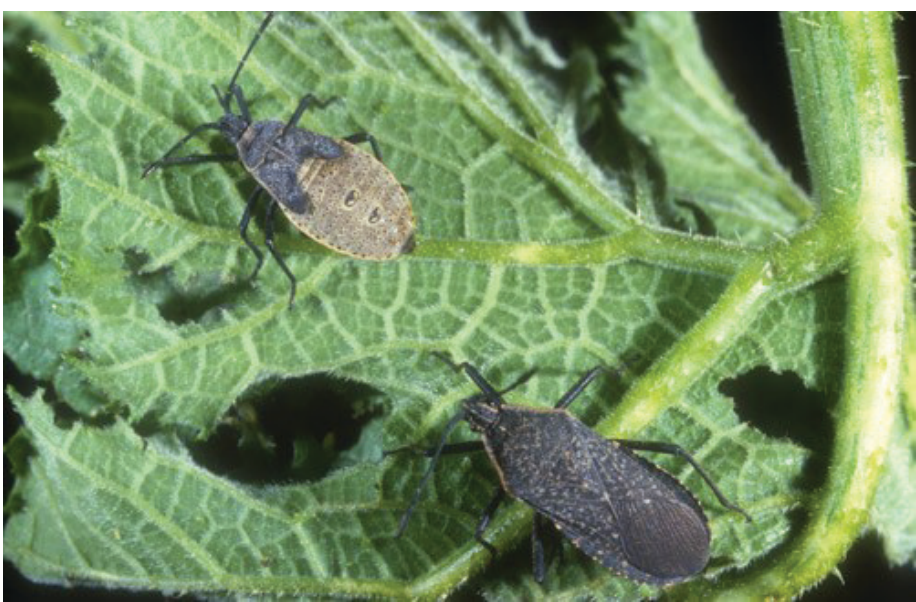

Figure 24. Adult (bottom) and nymph (top) squash bug, Anasa tristis. Credits: John L. Capinera, UF/IFAS
Sweet Potato Weevil

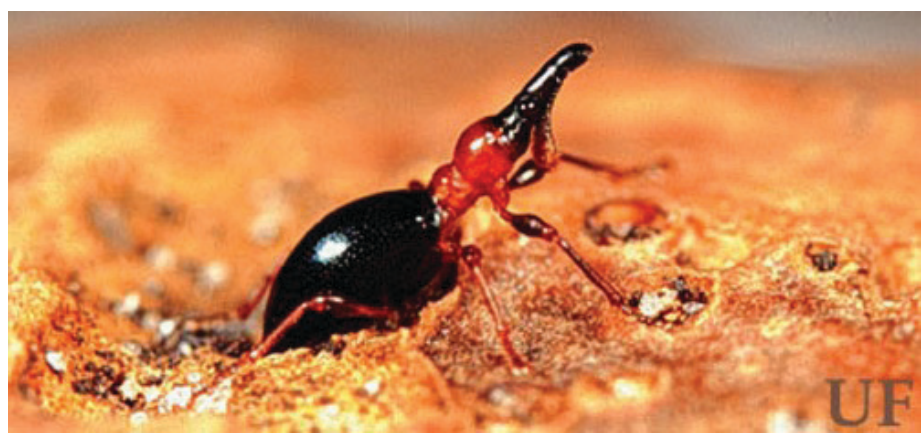

Figure 25. Adult sweet potato weevil, Cylas formicarius.

Credits: James Castner, UF/IFAS

Tomato Hornworm

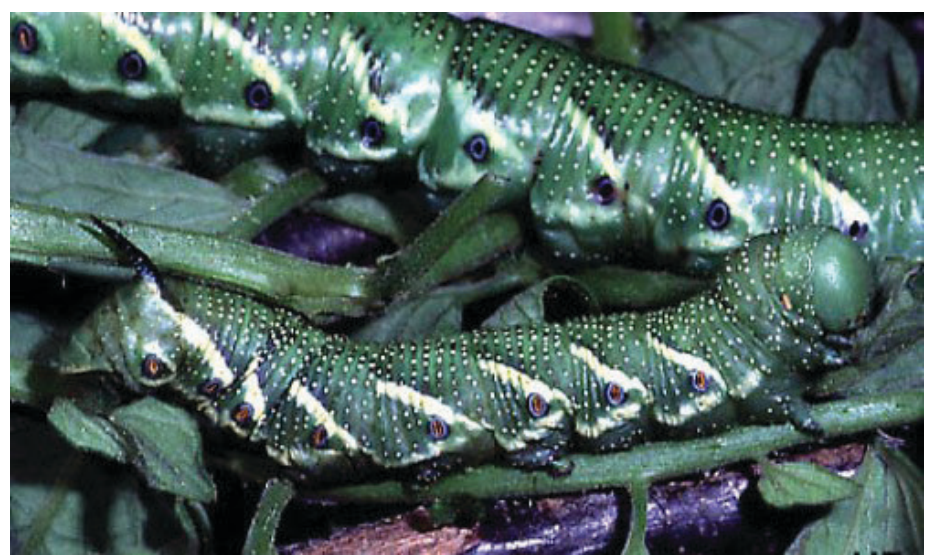

Figure 26. Last instar larva of the tomato hornworm, Manduca quinquemaculata.

Credits: Paul M. Choate, UF/IFAS

Twelve-Spotted Cucumber Beetle

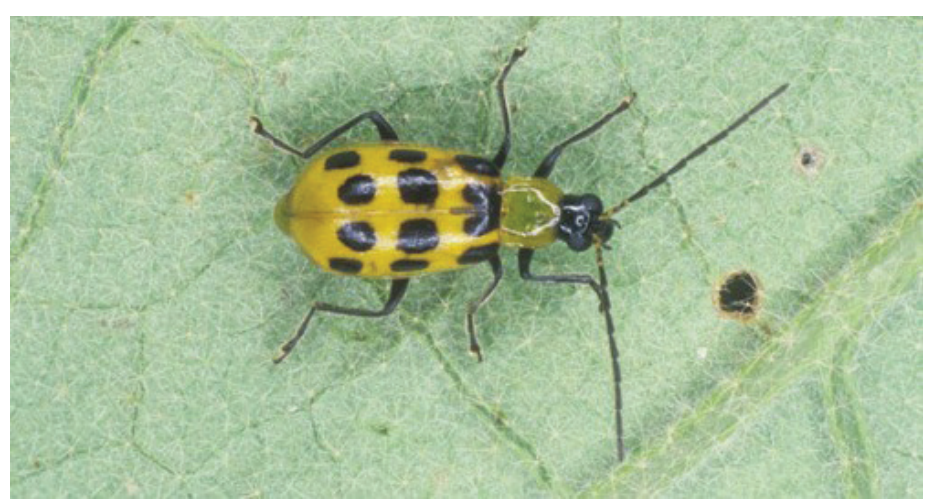

Figure 27. The spotted cucumber beetle, Diabrotica undecimpunctata howardi.

Credits: James Castner, UF/IFAS 
White Grub

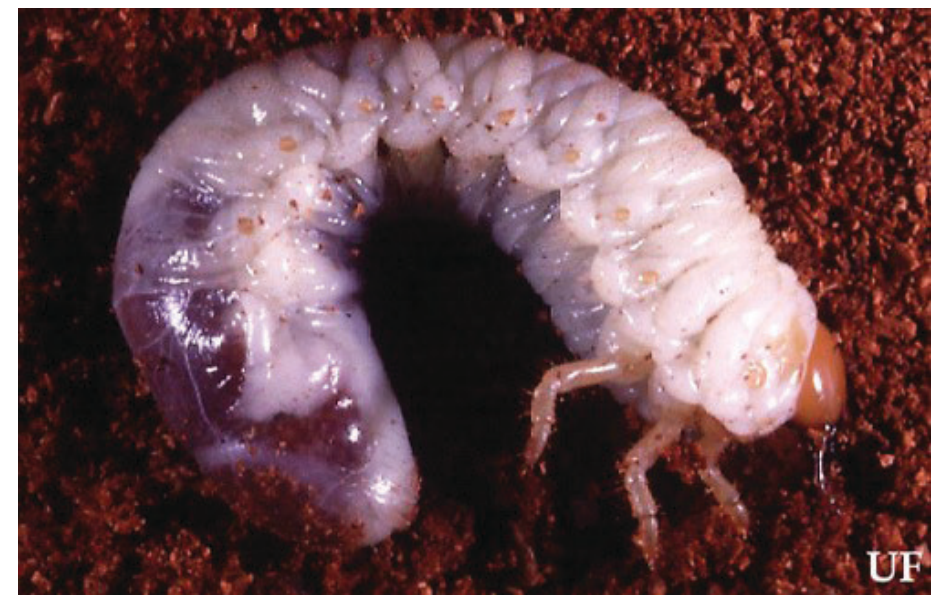

Figure 28. Typical white grub of the genus Phyllophaga.

Credits: John L. Capinera, UF/IFAS

Wireworm

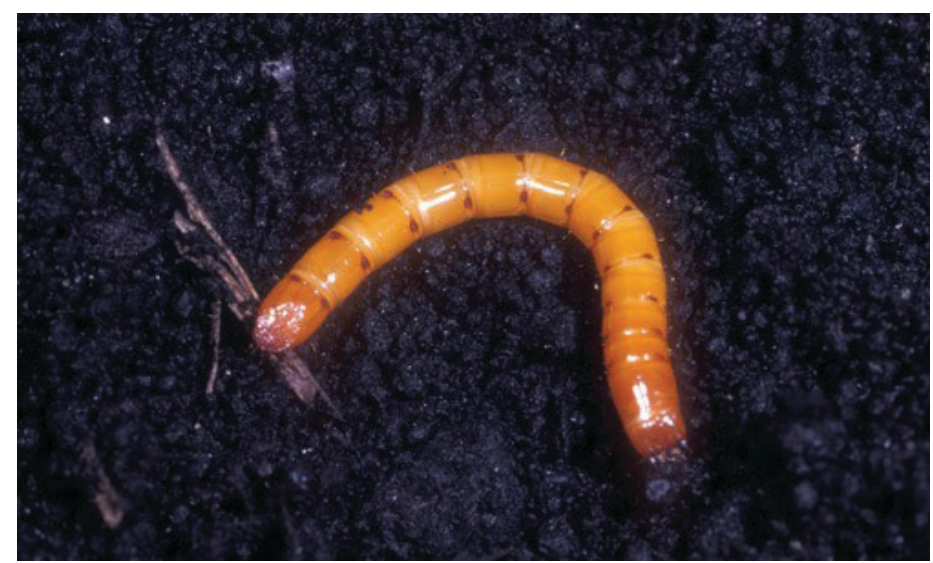

Figure 29. Larva of Melanotus communis, also known as a wireworm. Credits: Lyle Buss, UF/IFAS

\section{Beneficials}

Earwig

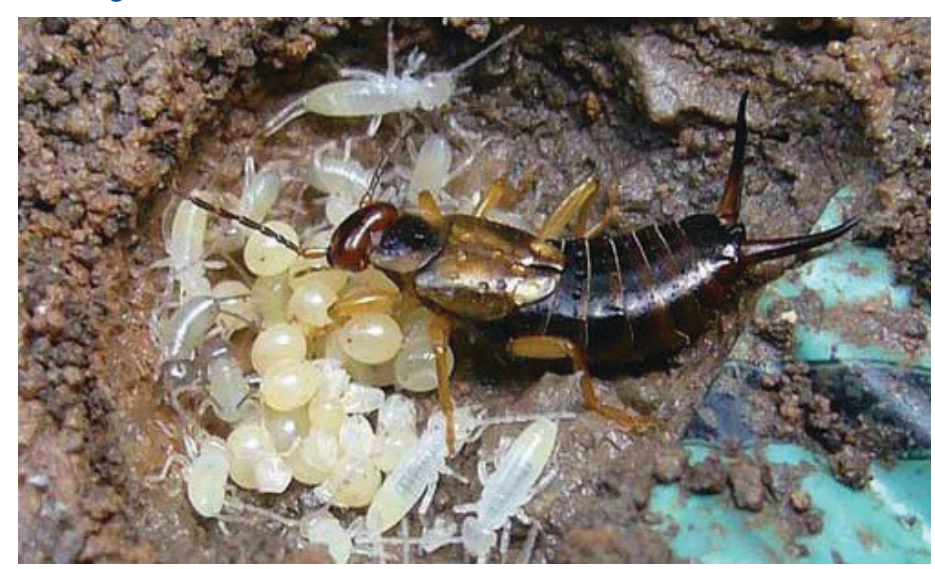

Figure 30. Adult female European earwig, Forficula auricularia, with eggs and young.

Credits: Nabokov, en.wikipedia.org, Chester, United Kingdom
Green Lynx Spider

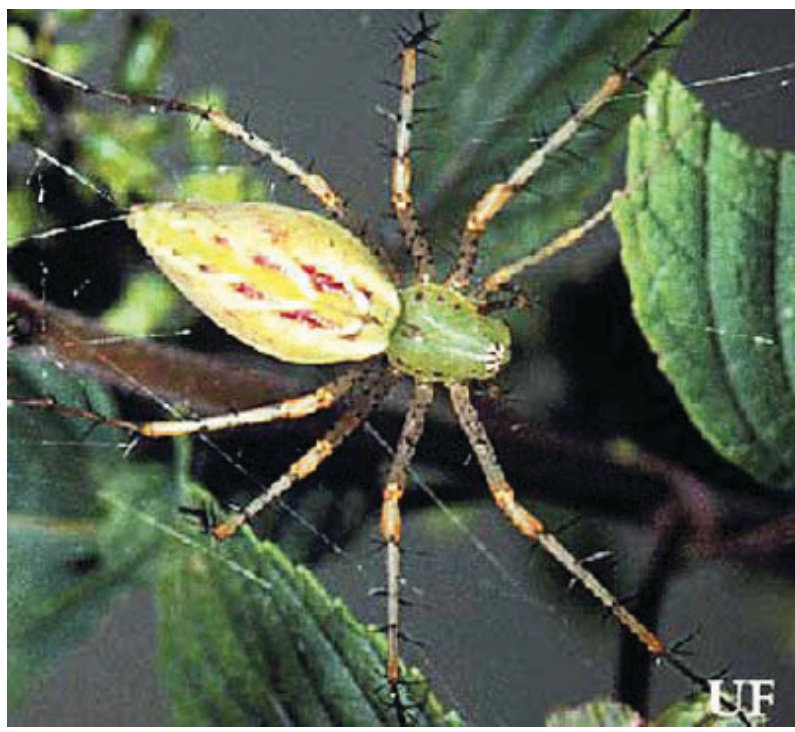

Figure 31. Adult green lynx spider, Peucetia viridans.

Credits: UF/IFAS

Ground Beetle

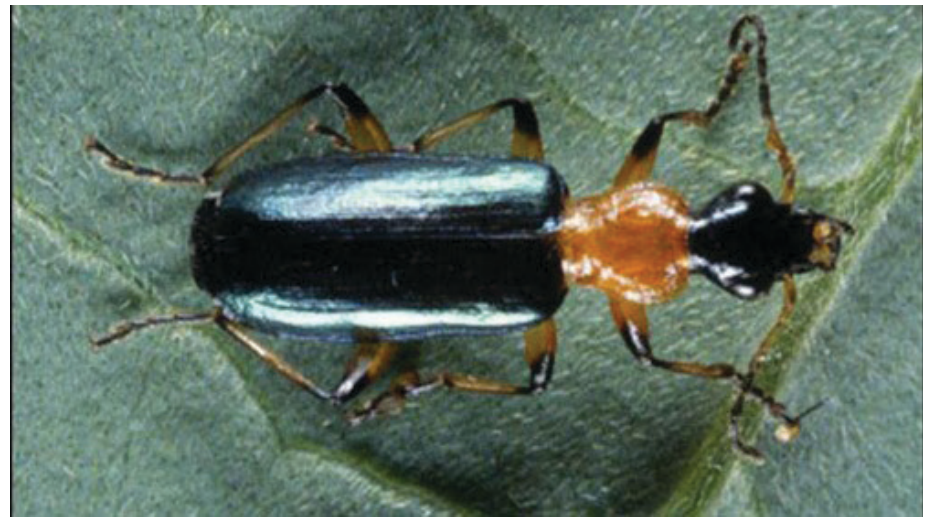

Figure 32. Adult Calleida decora, a ground beetle.

Credits: Lyle J. Buss, UF/IFAS

Honey Bee

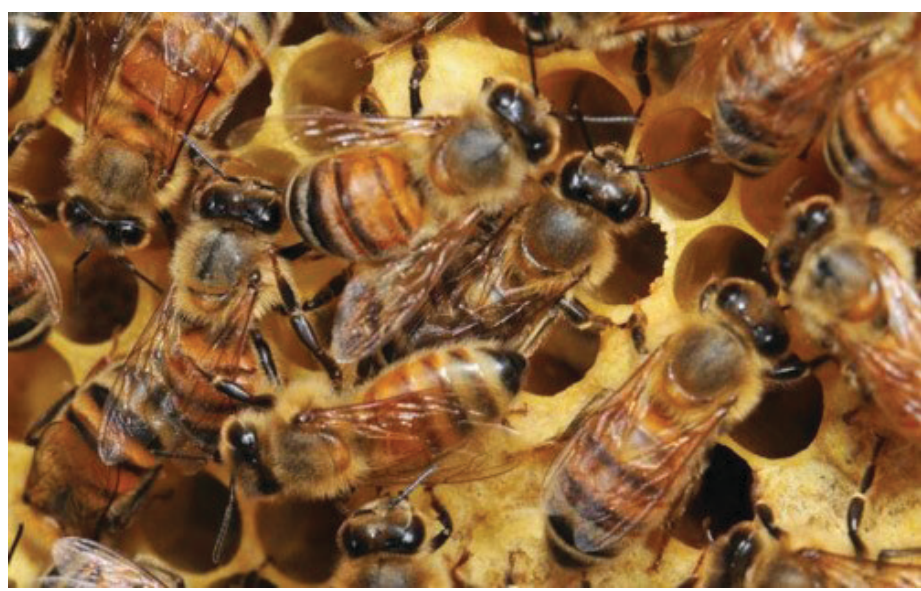

Figure 33. European honey bees, Apis mellifera, on a comb in a colony. Credits: Ashley N. Mortensen, UF/IFAS 
Lady Beetle

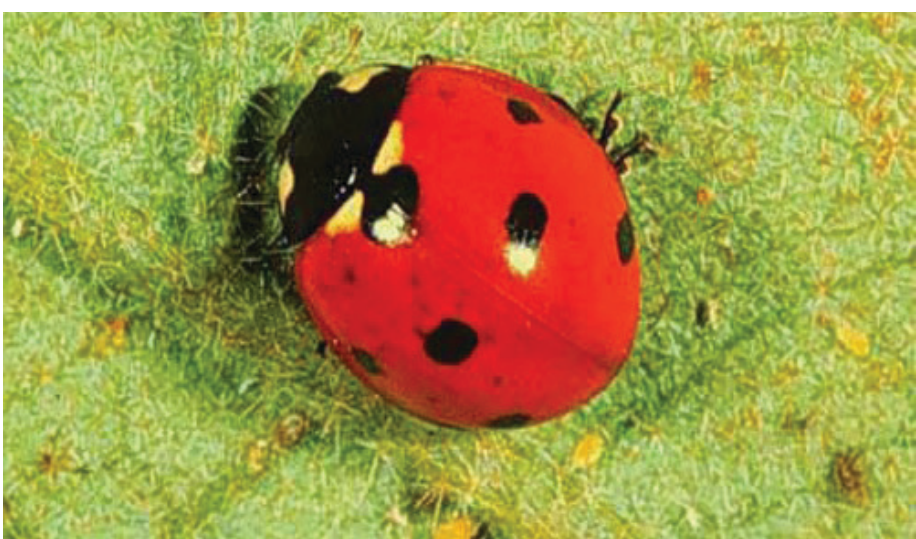

Figure 34. Adult Coccinella septempunctata, the seven-spotted lady beetle.

Credits: James Castner, UF/IFAS

Praying Mantis

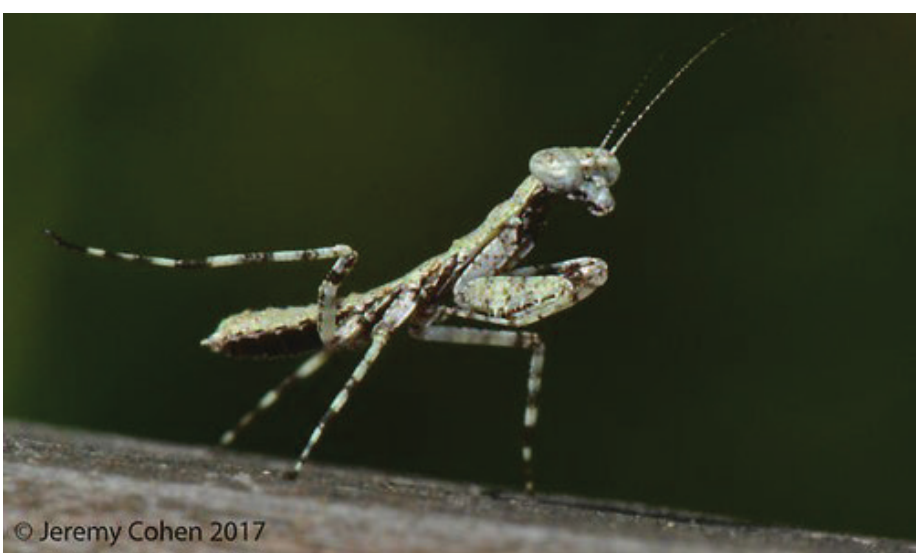

Figure 35. Grizzled mantid, Gonatista grisea (Fabricius), nymph. Credits: Jeremy Cohen, University of South Florida

*Tachnid Fly (links to BugGuide)

Tiger Beetle

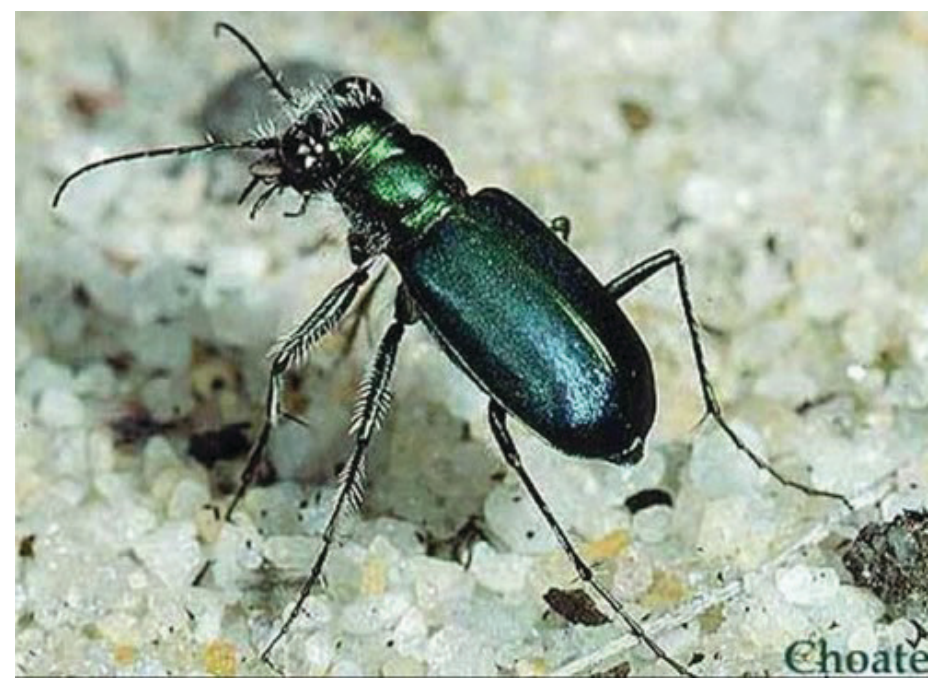

Figure 36. A tiger beetle, Cicindela scutellaris unicolor.

Credits: Paul M. Choate, UF/IFAS
We will update links as new articles are added to the Featured Creatures database.

Special thanks to the original authors of the publications linked here and to the photographers for sharing their photos. The photographers retain their rights to the images used here. Please contact them if you would like to copy or reproduce their photos. When possible, an email address for a photographer has been linked to their name. 